\title{
A lookup table-based method for wheel-rail contact analysis
}

\author{
M Bozzone, E Pennestrì*, and P Salvini \\ Department of Mechanical Engineering, University of Rome ‘Tor Vergata', Rome, Italy
}

The manuscript was received on 21 September 2010 and was accepted after revision for publication on 29 November 2010.

DOI: $10.1177 / 2041306810394721$

\begin{abstract}
This article describes an investigation on the relative motion between wheel-set and rails. Through the search of an equilibrium configuration, the positions of contact points between rails and wheels are first located. The detection methods allow also the definition of the normal unit vectors to rail and wheel at contact points. To reduce computing time, the results are stored in a lookup table accessed during the dynamic analyses of wheel-sets, bogies, or trains. The use of lookup table considerably speeds up contact detection when numerically integrating with respect to time.
\end{abstract}

Keywords: dynamics, contact analysis, wheel-rail interaction, wheel-set

\section{INTRODUCTION}

The solution of wheel-rail contact problem is required by the dynamic analysis of railways systems. Since most of the computer time is spent on contact search, this phase is crucial in all the available computer codes [1]. Many different approaches have been proposed that overcome the simple assumption of rigid contact, by introducing appropriate contact stiffness evaluations. The method proposed by Sanborn et al. [2], based on the numerical solution of index1 sparse differential-algebraic system of equations, requires online contact search and analysis. Their approach, although accurate, appears computationally very intensive. The method herein proposed is based on the numerical integration of a system of Ordinary Differential Equations that offer better numerical performances. Moreover, off-line contact search, although less accurate, allows considerable savings of CPU time. These issues are significant for the simulation of an entire train convoy.

In reference [3], there is a brief but exhaustive synthesis of the so-called on-line and off-line contact search. The method herein proposed is based on the off-line approach. According to this criterion, a

\footnotetext{
* Corresponding author: Department of Mechanical Engineering, University of Rome 'TorVergata', Via del Politechnico, 1, Rome 00133, Italy.
}

email:pennestri@mec.uniroma2.it contact lookup table is preliminarily compiled before numerical integration of the equations of motion.

For each wheel-set and rail coupling it is necessary to define the spatial attitude of the axle in its feasible workspace, to locate the actual wheel-rail contact points and to compute the value of the normal force components.

The compilation of a lookup table - where the numeric relationships between co-ordinates are stored - makes possible the development of a computationally efficient software dedicated to the dynamic simulation of the wheel-set, the bogie, or, more generally, the entire railway convoy. Thus, different types of analyses [4-8] can be carried out, such as the determination of wheel-set critical speed and the computation of contact forces under dynamic conditions.

During the dynamic analysis, the implemented software solves numerically the equations of motion. At each time step the dependent coordinates, consistent with the contact constraints, are obtained through interpolation from the lookup table.

The advantages of the methods herein presented are:

(a) reduction in the computation time, since the identification of the contact conditions is obtained by a linear interpolation scheme;

(b) possibility to extend the analysis over a long time interval;

(c) capability of a real-time simulation of an entire convoy with the appropriate hardware; 
(d) possibility to study the dynamics with different wheel-set and rail profiles through an update of the lookup table.

Different methodologies for the investigation on wheel-rail contact are available [9-14]. These can be classified into two main groups. The first group searches for the contact points by solving the system of algebraic-differential equations that includes the constraints due to rigid contact between wheels and rails. The second group, to which the methods herein presented belongs, introduces elasticity between the mating surfaces.

In reference [9], the contact points are determined through the concept of difference surface, defined as the distance - measured along the normal direction between the wheel and rail surfaces.

If such difference surface has only positive values, then the rail and the wheel are not in contact. If the difference surface shows at least some negative values, then there are one or more contact areas. Contact points are located where the difference surface reaches its local minima, with a negative value of the function. Accordingly, at these points the indentation between the contact bodies attains a maximum. This criterion is also used to estimate the contact force components. In order to calculate the minimum of the difference, the simplex method is iteratively exploited.

The results obtained using this method are compared with those achieved using the grid method. This last evaluates the function in a series of points and finds the minima comparing all obtained values. The reliability of the grid method depends mainly on the number of points chosen to evaluate the function. Even if the grid method is conceptually simple and reliable, it cannot be used for this type of application because of its high computational cost.

In references [10] to [12], a method alternative to those based on lookup tables is considered. The contact characteristics are calculated on-line during the dynamic simulation, considering the sections of the rails and the wheels for each feasible position of the wheel-set on the rails. For each section a group of nodal points is used through spline interpolation to mimic the profiles of the contact surfaces. The position of the contact points and directions of normal unit vectors are then calculated. In particular, it is required that the vector joining the contact points, one on the rail and the other on the wheel, and the vector normals through these points, belong to the same straight line. This method allows the online change of surface profiles. This feature is useful in case of rail-switch, local gauge, and slope variations.

In references [13] to [16], two methods for the determination of the contact points are presented: the constraint method and the elastic method, with which the normal forces on the surfaces can be calculated as well.

In the constraint method, the contact points on both solids coincide since the kinematic contact constraint is enforced. In the elastic method the contact points do not, in general, coincide. The contact point on the first body is located inside the volume of the second body when the solids interact with each other. In this case, the contact points are selected within the intersecting volume. Points presenting the maximum normal distance are the elected contact points.

Since in the constraint method the surfaces are rigid, no penetration is allowed between the bodies. On the other hand, in the elastic method penetration occurs and this is used to evaluate the contact forces. These are null when penetration is absent. Two surface parameters are used to describe the geometry of each of the two surfaces in contact. In the analysis, the contact points are grouped in batches. A batch is a collection of sets of pairs of nodal points on the wheel and rail, respectively. A limit of two contact batches is assumed. The two points (one on the wheel and one on the rail) that lead to the maximum indentation are selected as the points of contact for any given batch. The number of points of contact between the wheel and the rail is assumed to be equal to the number of the contact batches.

All methods share the difficulties of lengthy computing time and of managing great amounts of data. In fact, these analyses usually require many iterations and the matrices containing the surface geometries are huge.

In this article, three new different methods for the compilation of a lookup table, storing precalculated solutions for the wheel-rail contact kinematics, are discussed. According to this point of view, the location of contact points is preliminarily established for a given finite set of independent variables. In a dynamic analysis, the values of the dependent variables are obtained through interpolation on a discrete set of values. The accuracy of the interpolation depends on the different factors such as the Euclidean distance between two adjacent nodes of the mesh, the local linearized shape of the mesh, and the admissible penetrating gap. The numerical tests carried out herein confirm the reliability and the computational efficiency of the method.

Although the authors' software was running in a non-compiled version, CPU times are comparable with those required by commercial codes.

\section{THE WHEEL-SET-RAILS SYSTEM}

The shape profile of the wheels and of the rails section are $\$ 1002$ and UIC60, respectively. Rail tilt is set to $1 / 20$. The wheel-set has six degrees of freedom when not 


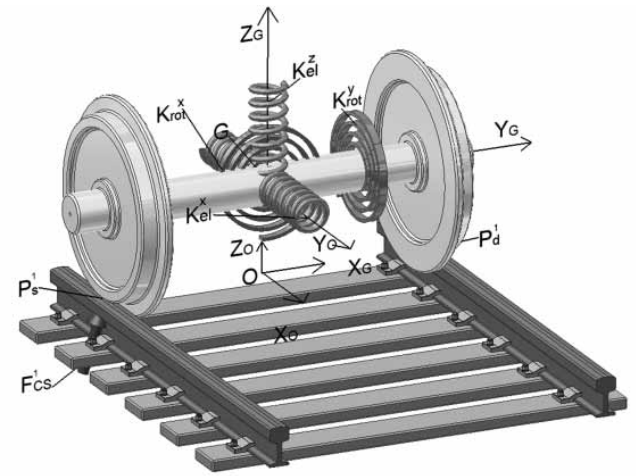

(a)

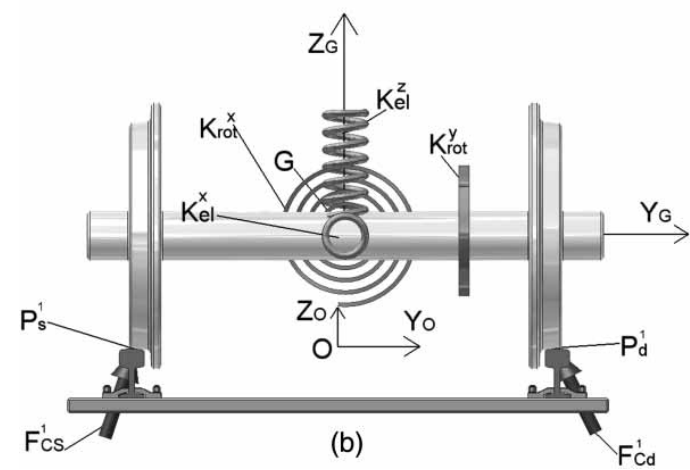

Fig. 1 The wheel-set, the rails and the fictitious spring elements with the Cartesian coordinate systems used: (a) axonometric view and (b) frontal view

constrained. These degrees of freedom reduce to three due to the constraints introduced by imposing continuous contact with rails. However, the wheel rotation has no effect on the contact kinematics. Thus, only two independent variables need to be prescribed to compute the spatial position of the wheel-set.

With reference to Fig. 1, a fixed and a moving righthand Cartesian coordinate system are introduced. The fixed inertial coordinate system, denoted by SRo, has its origin $\mathrm{O}$ in the middle of the gauge, the axis $\mathrm{X}_{\mathrm{o}}$ tangent to the rails, and the $Z_{0}$ axis directed upwards. The moving coordinate system, denoted by SRG, has its origin in the centre of mass $G$ of the wheel-set, axis $Y_{\mathrm{G}}$ directed along the axis of the axle, and the $Z_{\mathrm{G}}$ axis directed upwards.

Initially the axes of the fixed and moving coordinate systems are all parallel to each other and the centre of mass $G$ is on $\mathrm{Z}_{\mathrm{o}}$ axis at a distance $r_{\mathrm{w}}$ from the origin $\mathrm{O}$.

The absolute coordinates of $G$ are denoted by $x_{g}, y_{g}$, $z_{g}$ whereas $\theta_{x}, \theta_{y}, \theta_{z}$ denote the angles between couples of $x, y$, and $z$ axes, respectively.

The displacement $y_{g}$ and the yaw angle $\theta_{z}$ are assumed as independent variables; therefore, the three dependent variables $\left(x_{g}, z_{g}, \theta_{x}\right)$ are deduced by imposing a continuous physical contact between rails and wheel-set. The sixth variable $\left(\theta_{y}\right)$ follows from dynamic equilibrium.

\section{MODEL SET-UP}

The first step of the modelling is the acquisition of wheel and rail profiles, as shown in Fig. 2. The surface of the rail is obtained by the extrusion of the rail profile along the $X_{\mathrm{o}}$ axis.

The surface of the wheel is generated through a revolution of the wheel profile about $Y_{\mathrm{G}}$, as shown in Fig. 3.

The two surfaces are meshed. The coarser the mesh, the less the expected accuracy and the computation time.

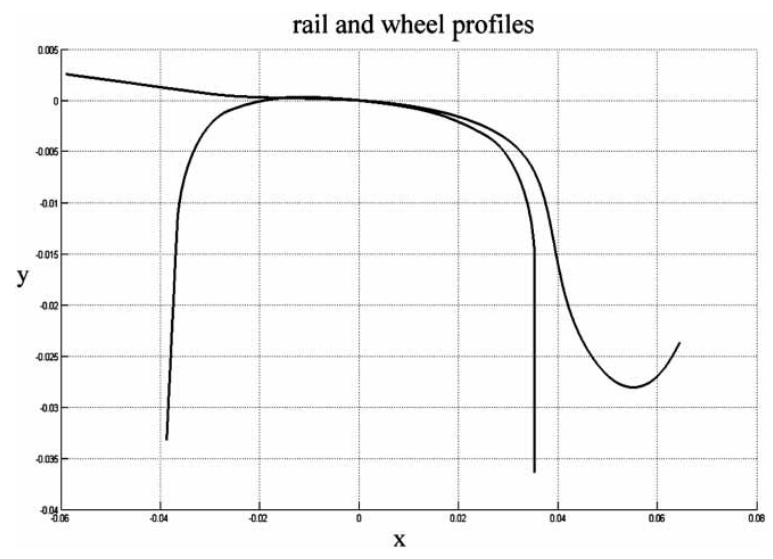

Fig. 2 Wheel and rail shape profile

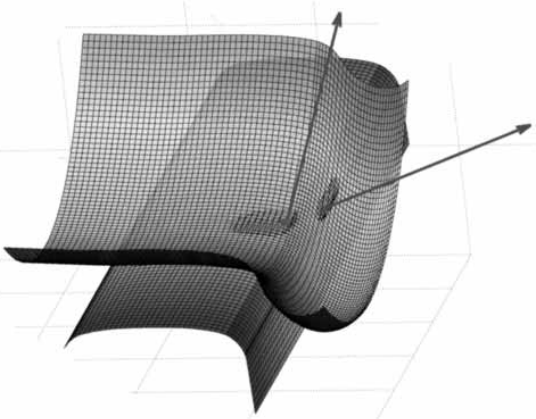

(a)

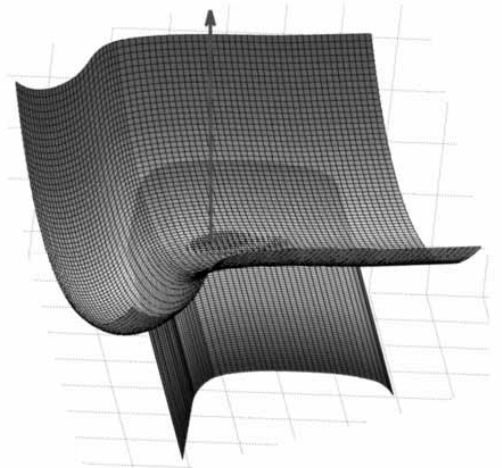

(b)

Fig. 3 Wheel and rail surfaces in the condition of: (a) double contact and (b) single contact 
In spite of locating the contact points, as shown in Fig. 1, four fictitious spring elements are added to the wheel-set: three for each dependent position variable and one to avoid lability. The springs ends are attached to $\mathrm{G}$ and to ground, respectively. Both linear $(\mathrm{N} \mathrm{m})$ and torsion spring $(\mathrm{N} \mathrm{m})$ stiffnesses are numerically set to $10^{7}$. Under static conditions the forces and torques generated by spring elements and the contact forces satisfy the following vector equilibrium equations

$$
\begin{aligned}
& \sum_{i=1}^{3} \boldsymbol{k}_{\mathrm{el}}^{i} x_{\mathrm{Gi}} \boldsymbol{H}_{i}+\sum_{h=1}^{n_{\mathrm{CL}}} \boldsymbol{F}_{\mathrm{cL}}^{h}+\sum_{g=1}^{n_{\mathrm{CR}}} \boldsymbol{F}_{\mathrm{cR}}^{g}=0 \\
& \sum_{i=1}^{3} k_{\mathrm{rot}}^{i} \theta_{i} \boldsymbol{H}_{i}+\sum_{h=1}^{n_{\mathrm{CL}}}\left(\boldsymbol{G} \boldsymbol{P}_{L}^{h} \times \boldsymbol{F}_{\mathrm{cL}}^{h}\right) \\
& +\sum_{g=1}^{n_{\mathrm{CR}}}\left(\boldsymbol{G}_{R}^{g} \times \boldsymbol{F}_{\mathrm{cR}}^{g}\right)=0
\end{aligned}
$$

\section{CONTACT POINTS DETECTION}

\subsection{The intersecting volume}

As shown in the geometry depicted in Fig. 4, the intersecting volume $V_{\text {int }}$ is the one shared by the wheel and rail when they contact. Let $S_{\mathrm{w}}$ and $S_{\mathrm{r}}$ denote the wheel and rail portions of surface delimited by $n_{\mathrm{w}}$ and $n_{\mathrm{r}}$ nodes, respectively, and $i=3, \ldots, n_{\mathrm{w}}$ and $j=3, \ldots, n_{\mathrm{r}}$ the nodes of the wheel and rail surfaces, respectively.

The $i$ th node and $j$ th node are included in the intersecting volume only if the following inequalities are simultaneously satisfied

$$
\begin{aligned}
& \boldsymbol{V}_{i j} \cdot \boldsymbol{N}_{j} \geqslant 0 \\
& \boldsymbol{V}_{i j} \cdot \boldsymbol{N}_{i} \leqslant 0
\end{aligned}
$$

Among the nodes included in the intersecting volume, those which belong to the wheel or to the surface $S_{\mathrm{w}}$ and form the intersecting volume $V_{\text {int }}^{\mathrm{w}}$ are distinguished from those which belong to the rail or to the surface $S_{\mathrm{w}}$ and form the volume $V_{\mathrm{int}}^{\mathrm{r}}$. The union of $V_{\text {int }}^{\mathrm{w}}$ and $V_{\text {int }}^{\mathrm{r}}$ gives the volume $V_{\text {int }}$. Thereafter, attention is focused on the determination of the centre of contact on both surfaces. However, intersecting volume magnitudes are not really necessary. This is the reason why nodal point coordinates are used to test contact conditions.

\subsection{The contact points}

Three different methods for computing wheel-rail contact points are proposed and discussed. All the methods share the same objective of locating the intersections between $V_{\mathrm{int}}^{\mathrm{w}}$ and $V_{\mathrm{int}}^{\mathrm{r}}$. These methods are termed as follows:

(a) maximum distance;

(b) maximum normal distance;

(c) load centre.

\subsubsection{Method of the maximum distance}

All nodes included in $V_{\text {int }}$ are subject to be contact points between the surfaces. For each node $i^{*}$ of $S_{\mathrm{w}}$ (within $V_{\text {int }}$ ) it is found the node $j^{*}$ of $S_{\text {r }}$ (within $V_{\text {int }}$ ) such that the norm of $\boldsymbol{V}_{i^{*} j^{*}}$ has the minimum value. In this way, a correspondence between the set of nodes $i^{*}$ and the set of nodes $j^{*}$ is established. The indentation is computed by means of the scalar product

$$
C_{i^{*} j^{*}}=\boldsymbol{V}_{i^{*} j^{*}} \cdot \boldsymbol{N}_{j^{*}}
$$

Among all the set of nodes $i^{*}, j^{*}$ the contact points are those experiencing the maximum value of $C_{i^{*} j^{*}}$

$$
C_{\max }=\max \left(C_{i^{*} j^{*}}\right)
$$

\subsubsection{Method of the maximum normal distance}

This method offers a refinement in the computation of contact point $P_{\mathrm{r}}^{\prime}$ but needs the election of a reference surface. Here the wheel surface is adopted.

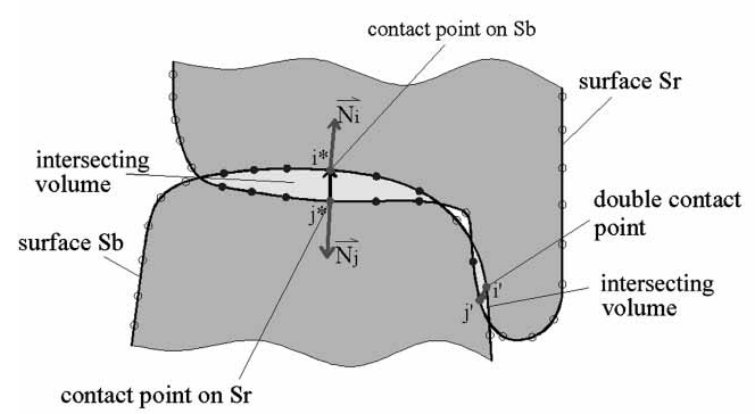

(a)

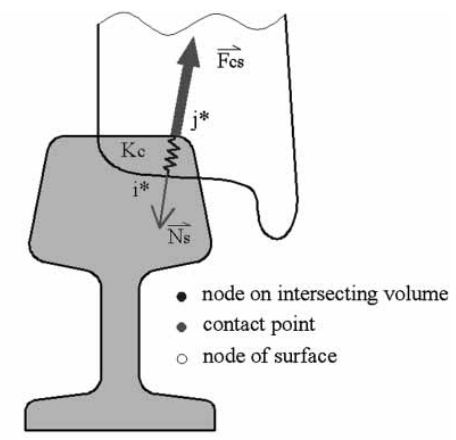

(b)

Fig. 4 (a) Wheel-rail intersecting volume according to the method of maximum distance;

(b) scheme of the elastic contact force 


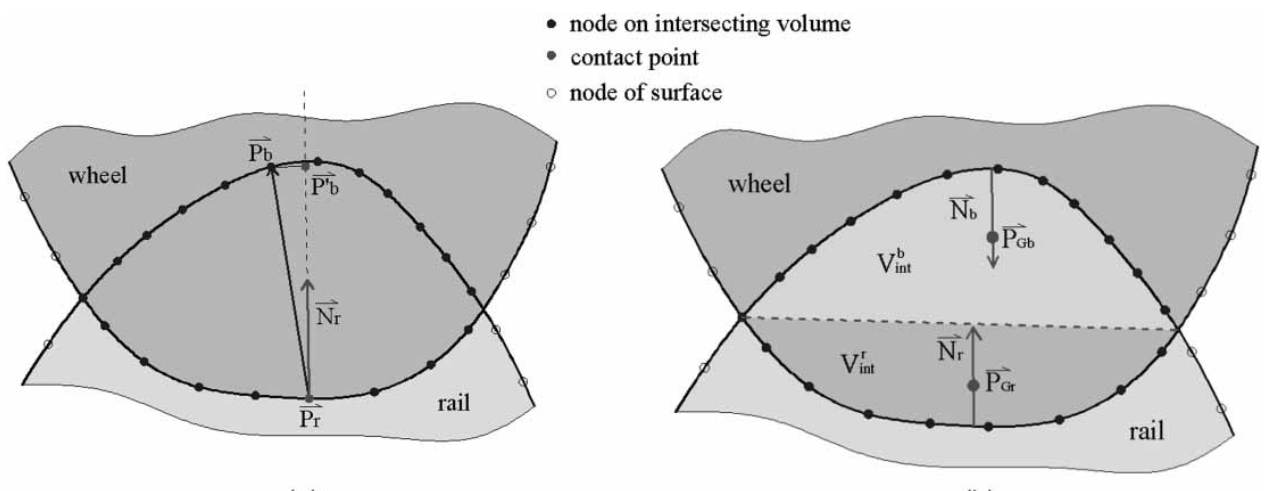

(a)

(b)

Fig. 5 Wheel-rail intersecting volume according to: (a) the method of the maximum normal distance, (b) method of the centre of load

The method of maximum distance is initially applied. Then, with reference to the geometry depicted in Fig. 5(a), the coordinates of the new contact point $P_{\mathrm{r}}^{\prime}$ on the rail are obtained from

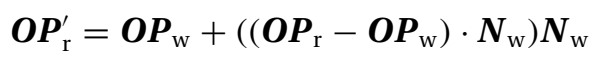

The maximum indentation $C_{\max }$ is calculated using the formula

$$
C_{\max }=\left(\boldsymbol{O P}_{\mathrm{r}}-\boldsymbol{O P}_{\mathrm{w}}\right) \cdot \boldsymbol{N}_{\mathrm{w}}
$$

With this method the accuracy of computation is less dependent on the surface mesh coarseness. This turns also into an overall speedup. The main limitation of this method is that the contact points on the wheel surface need to be located on the grid nodes.

\subsubsection{Method of the centre of load}

In this method the volumes of intersection $V_{\text {int }}^{\mathrm{r}}$ and $V_{\text {int }}^{\mathrm{w}}$ are separately considered (Fig. 5(b)).

The contact points are made coincident with the load centre $P_{\mathrm{Gw}}$ and $P_{\mathrm{Gr}}$ of the grid nodes on the boundary of $V_{\text {int }}$. Analytically this leads to

$$
\boldsymbol{O P}_{\mathrm{Gw}}=\frac{\sum_{i=1}^{n_{\mathrm{w}}} \boldsymbol{O} \boldsymbol{P}_{i}^{\mathrm{w}}}{n_{\mathrm{w}}} ; \quad \boldsymbol{O P}_{\mathrm{Gr}}=\frac{\sum_{j=1}^{n_{\mathrm{r}}} \boldsymbol{O} \boldsymbol{P}_{j}^{\mathrm{r}}}{n_{\mathrm{r}}}
$$

The maximum indentation $C_{\max }$ is calculated by means of the formula

$$
C_{\max }=\left(\boldsymbol{O P} \boldsymbol{P}_{G r}-\boldsymbol{O P} \boldsymbol{P}_{G w}\right) \cdot \boldsymbol{N}_{\mathrm{w}}
$$

Since $P_{\mathrm{Gw}}$ does not belong to the wheel surface.

\subsection{Normal contact forces}

With reference to the geometry depicted in Fig. 4, the normal contact forces $\boldsymbol{F}_{\mathrm{cL}}$ and $\boldsymbol{F}_{\mathrm{cR}}$, in the left and right wheel-rail interface, respectively, are computed as follows

$$
\begin{aligned}
& \boldsymbol{F}_{\mathrm{cL}}=-C_{\text {max }}^{\mathrm{L}} k_{\mathrm{c}} \boldsymbol{N}_{\mathrm{L}} \\
& \boldsymbol{F}_{\mathrm{cR}}=-C_{\text {max }}^{\mathrm{R}} k_{\mathrm{c}} \boldsymbol{N}_{\mathrm{R}}
\end{aligned}
$$

The value of $k_{\mathrm{c}}$ is established through iteration. In particular, convergence is achieved when the variables $x_{g}, y_{g}, z_{g}, \theta_{x}, \theta_{y}, \theta_{z}$ simultaneously satisfy both the equilibrium equations (1) and the following inequalities

$$
\begin{aligned}
& C_{\max }^{\mathrm{L}} \leqslant C_{\max }^{\mathrm{amm}} \\
& C_{\max }^{\mathrm{R}} \leqslant C_{\max }^{\mathrm{amm}}
\end{aligned}
$$

where $C_{\max }^{\mathrm{amm}}$ is the maximum indentation allowed.

In this investigation $C_{\max }^{\mathrm{amm}}=0.01 \mathrm{~mm}$ is assumed. This last value is broadly justified by some qualitative considerations. The value should be lower than the expected local compression, as suggested by the Hertz theory. Furthermore, the mesh refinement should be carried out in such a way that edge effects due to meshing are lower than that assumed value.

It should be observed that the values of contact stiffness, within a given range, do not influence the position of the contact points which depends on the surface geometry. A changing of the stiffness values for $n$ times, with $n$ spanning between 1 and 10 , causes the maximum values of the contact point displacements to be less than $10^{-19} \mathrm{~m}$.

This result proves that the position of the contact point is almost independent of the initial preload of the external springs. This rests upon the convergence of the iterative method.

The proposed algorithm for the detection of contact points can be summarized in the following steps.

1. Prescribe the values of the independent variables $y_{g}$ and $\theta_{z}$. Set to zero the dependent variables $x_{g}, z_{g}$, $\theta_{x}, \theta_{y}$. 
2. Prescribe an initial value to the stiffness $k_{\mathrm{c}}$.

3. Define the intersecting volume $V_{\text {int }}$, the position of the provisory contact points, vector normals, and maximum indentation between the wheels and the rails.

4. Compute the normal forces using equations (3).

5. Update the values of $x_{g}, z_{g}, \theta_{x}, \theta_{y}$ using equilibrium equations (1).

6. Update the intersecting volume $V_{\text {int }}$, the position of the provisory contact points, vector normals, and maximum indentation between the wheels and the rails.

7. If the maximum indentation is less than $C_{\max }^{\mathrm{amm}}$, then store in a database the values of $x_{g}, y_{g}, z_{g}, \theta_{x}, \theta_{y}, \theta_{z}$, and the contact points;

8. If the previous condition is not satisfied, then increase the value of $k_{\mathrm{c}}$ and go back to step 4 .

\subsection{The maximum indentation value}

The limit $C_{\max }^{\mathrm{amm}}=0.01 \mathrm{~mm}$, adopted as the maximum indentation value, has been established considering Hertz theory for the contact cylinder-plane. This rough approximation of the contact is herein adopted only for the search of contact points. The actual computation of the contact forces is performed within the framework of Kalker's theory $[17,18]$.

The average vertical load estimated on each wheel is $P=75000 \mathrm{~N}$.

The width $L=5 \mathrm{~mm}$ of the cylinder has the same order of magnitude of the transverse dimension for the wheel-rail contact area.

The radius $R$ of the cylinder is the same as that of the wheel. The cylinder and plane are both in steel with Young's modulus $E=2.06 \times 10^{11} \mathrm{~N} / \mathrm{m}^{2}$ and Poisson's ratio $v=0.3$.

Hence, the maximum value of the indentation is

$$
\begin{aligned}
& C_{\max }^{\mathrm{amm}}=\frac{2 P K}{L}\left(1+\ln \left(\frac{4 L^{3}}{2 K P R}\right)\right) \approx 0.11 \mathrm{~mm} \\
& \text { where } K=\frac{1-v^{2}}{\pi E}
\end{aligned}
$$

\subsection{The stiffness of the lateral flange}

Because of the special shape of the wheel profile with the presence of a lateral flange, a linearly variable value of wheel-flange stiffness $k_{\mathrm{c}}$ is herein assumed. In particular, $k_{\mathrm{c}}$ is linearly interpolated only on the flange between a maximum value $K_{\max }$ at the flange base and a minimum value $K_{\min }$ at the flange tip.

Here the non-linearity of the function load displacement is not considered because a given (unit) value of the indentation is assumed, whatever is the point of contact.

The value of $K_{\max }$ at the base of the flange is estimated considering only the deformation due to

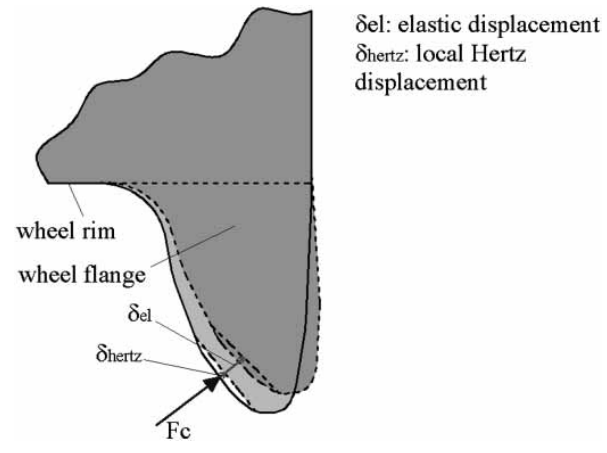

Fig. 6 Terms contributing to the total displacement of the flange

local Hertz approach. The value of $K_{\min }$ is calculated considering two contributions: the first one is the Hertz type of deformation as above and the second one regards the flange bending as a cantilever beam. Figure 6 illustrates the two contributions to the overall displacement caused by an applied force at the tip of the flange.

The stiffness, assuming Hertz theory and unitary indentation, is accounted by means of the formula [19]

$$
K_{\text {hertz }}=\frac{2 \pi G}{3(1-v)} \frac{1}{\sqrt{D_{\max }+D_{\min }}} \frac{\sqrt{E W(g)}}{g W(3)^{(3 / 2)}}
$$

where $D_{\max }$ and $D_{\min }$ depend on the local curvatures of the two surfaces in contact, where $D_{\max }=$ $\max \left(D_{\text {long }}, D_{\text {trasv }}\right), \quad D_{\min }=\min \left(D_{\text {long }}, D_{\text {trasv }}\right)$ assuming $D_{\text {long }}=(1 / 2)\left(C_{\text {long }}^{\mathrm{w}}+C_{\text {oong }}^{\mathrm{r}}\right)$ and $D_{\text {trasv }}=(1 / 2)\left(C_{\text {trasv }}^{\mathrm{w}}+\right.$ $\left.C_{\text {trasv }}^{\mathrm{r}}\right), C_{\text {long }}^{\mathrm{w}}$ and $C_{\text {long }}^{\mathrm{r}}$ are the longitudinal curvatures at contact point of the wheel and the rail, respectively; $C_{\text {trasv }}^{\mathrm{w}}$ and $C_{\text {trasv }}^{\mathrm{r}}$ are the transversal curvatures at contact point of the wheel and the rail, respectively; $g=\min ((a / b),(b / a))$ is a geometrical shape factor where $a$ and $b$ are the semi-axis lengths of the contact ellipse, $K(g)$ and $\mathrm{W}(g)$ are elliptic integrals, defined as follows

$$
\begin{aligned}
& W(g)=\int_{0}^{\pi / 2}\left[1-\left(1-g^{2}\right) \sin ^{2} \psi\right]^{-1 / 2} \mathrm{~d} \psi \\
& E(g)=\int_{0}^{\pi / 2}\left[1-\left(1-g^{2}\right) \sin ^{2} \psi\right]^{1 / 2} \mathrm{~d} \psi
\end{aligned}
$$

Replacing the values of the curvatures for the contact on the base and the tip of the flange, the stiffness values follow

$$
\begin{aligned}
& k_{\text {hertz }}^{\mathrm{bf}}=7.87 \times 10^{10}(\mathrm{~N} / \mathrm{m}) \\
& k_{\text {hertz }}^{\mathrm{tf}}=7.09 \times 10^{9}(\mathrm{~N} / \mathrm{m})
\end{aligned}
$$

where $k_{\text {hertz }}^{\mathrm{bf}}$ and $k_{\text {hertz }}^{\mathrm{tf}}$ are the values of the Hertz stiffness at the base and at the tip of the flange, respectively. The second contribution $k_{\mathrm{el}}^{\mathrm{tf}}$ is obtained considering the wheel flange as a cantilever beam 


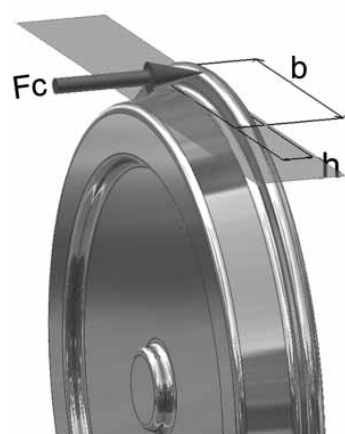

(a)

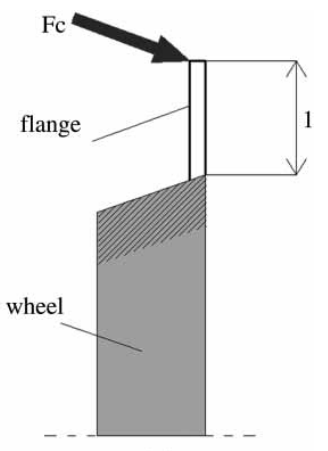

(b)
Fig. 7 Model of the flange as a cantilever beam: (a) Axonometric view and (b) schematic view

Table 1 Equivalent cantilever beam characteristics

\begin{tabular}{lll}
\hline$b=0.32 \mathrm{~m} \quad h=0.045 \mathrm{~m} \quad l=0.0275 \mathrm{~m} \quad \begin{aligned} k_{\mathrm{el}}^{\mathrm{tf}} & =\left(b h^{3} E / 4 l^{3}\right) \\
& =7.6 \times 10^{10}(\mathrm{~N} / \mathrm{m})\end{aligned}$ \\
\end{tabular}

(Fig. 7) whose dimensions are given in Table 1. The lateral dimension $b$ is such that the compliance of the cantilever beam is almost equal to the flange bending compliance, according to an appropriate static FE model, as discussed in reference [8].

The overall stiffness at the tip flange is

$$
k^{\mathrm{tf}}=\frac{k_{\text {hertz }}^{\mathrm{tf}} k_{\mathrm{el}}^{\mathrm{tf}}}{k_{\text {hertzx }}^{\mathrm{tf}}+k_{\mathrm{el}}^{\mathrm{tf}}}=6.48 \times 10^{9}(\mathrm{~N} / \mathrm{m})
$$

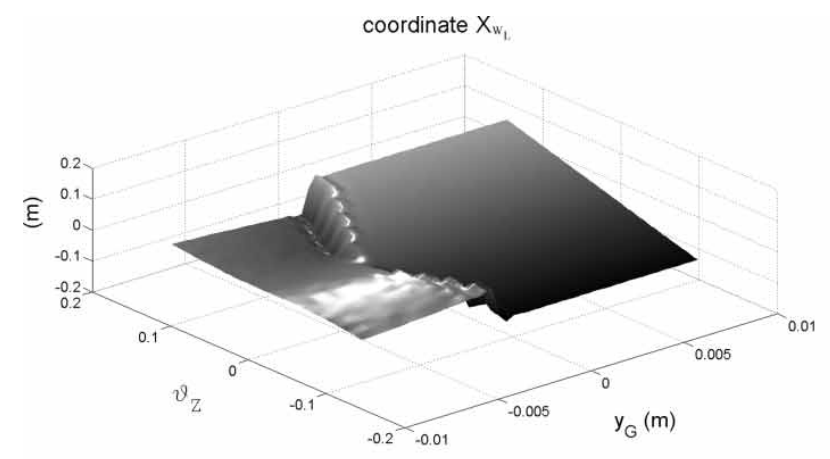

(a)

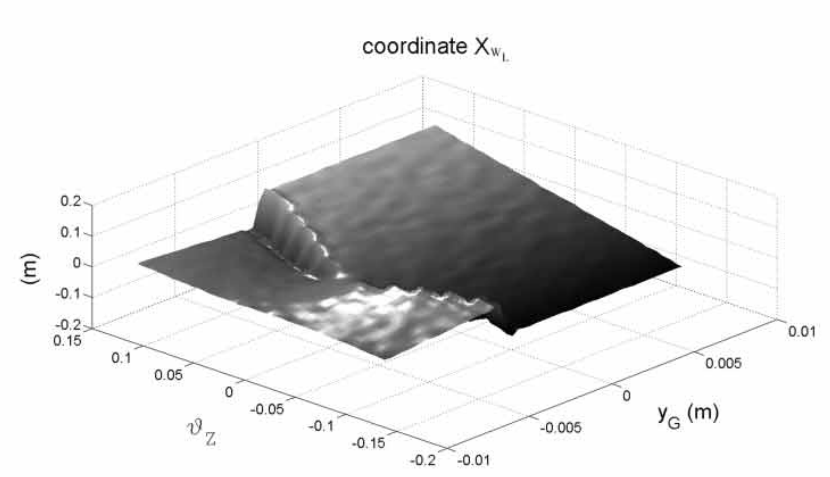

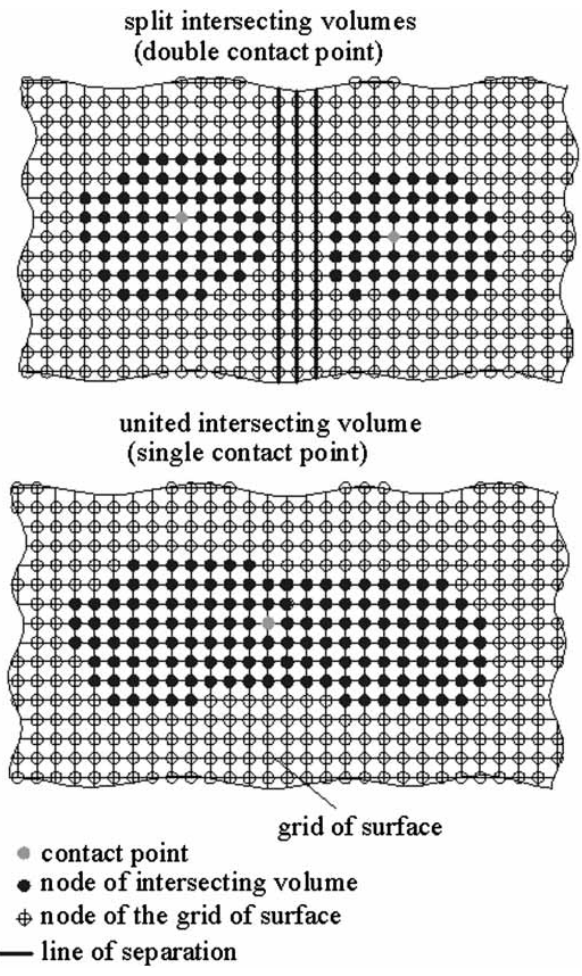

Fig. 8 Example of merged and split volumes of intersection

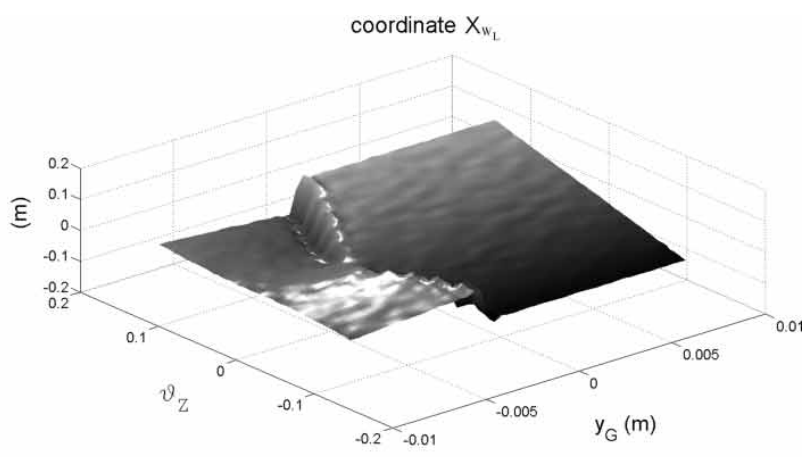

(b)

(c)

Fig. 9 Plots of $x$ coordinate of the contact point on the left wheel: (a) method of centre of load; (b) method of the maximum distance, and (c) method of the maximum normal distance 
whereas the ratio between the stiffnesses at the tip and the base of the flange is given by

$$
\chi=\frac{k^{\mathrm{tf}}}{k_{\text {hertz }}^{\mathrm{bf}}}=0.082
$$

The magnitude of this ratio, although significant, seems neglected in the models available in the current literature.

The intermediate stiffness values, within the base and the tip of the flange, are computed adopting a linear interpolation that assumes, as interpolating variable, the distance between the contact point and the axis of the wheel.

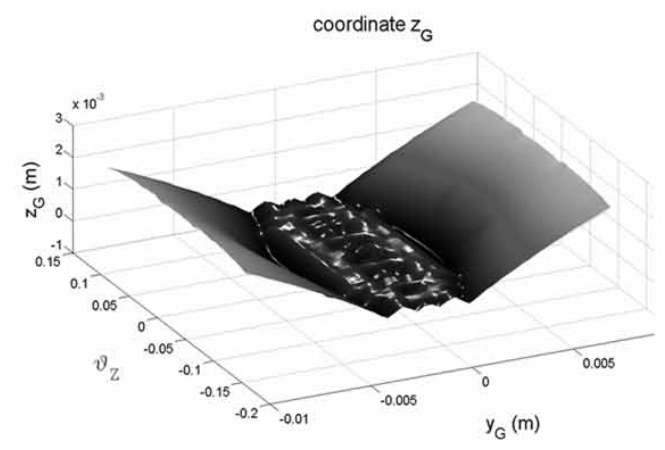

(a)

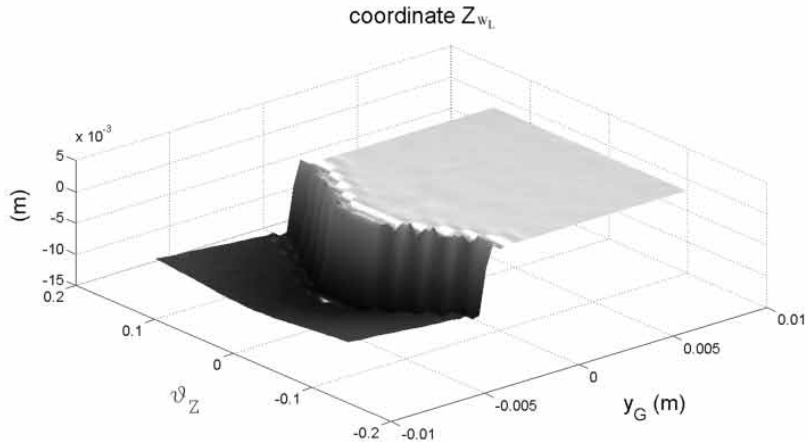

(c)

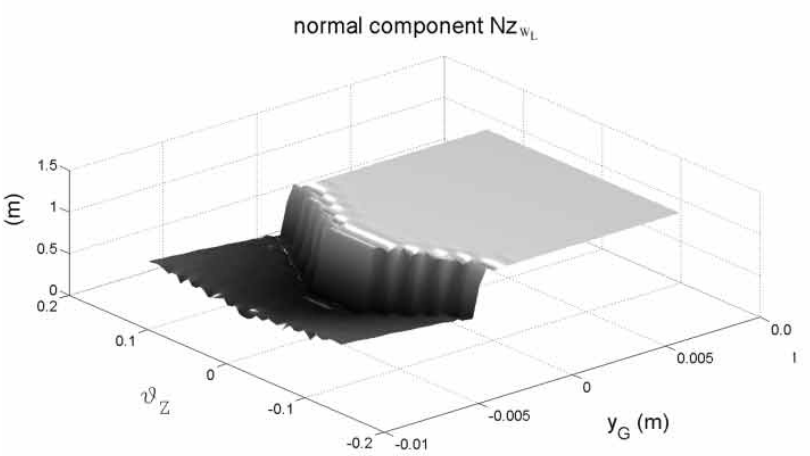

(e)
Finally, it should be observed that equation (11) is not used for computation of the wheel-rail contact forces during the dynamic analysis. Its use is only limited to the compilation of the lookup table. The numerical experience confirms that the linearization herein introduced on the computation of Hertz stiffness does not significantly affect the double-contact search.

\subsection{The double contact}

In most cases, our model finds only one point of contact between the wheel and rail surfaces.

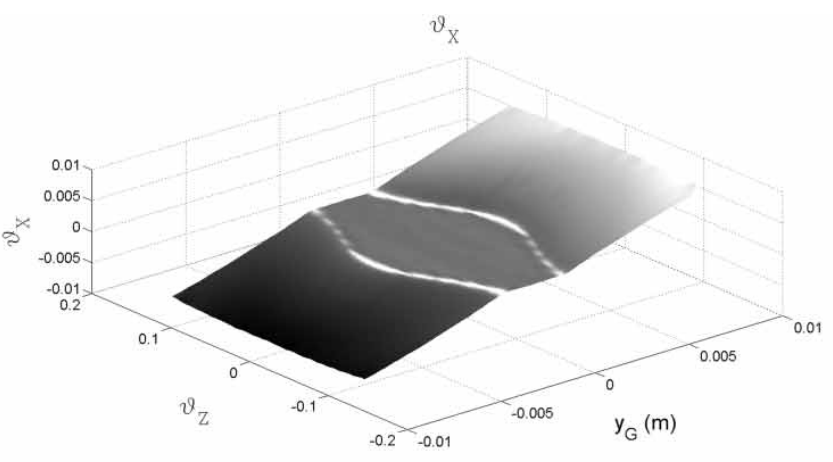

(b)

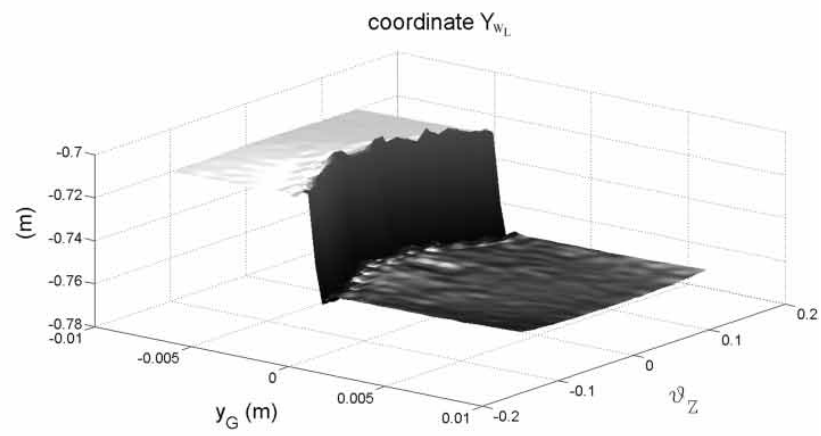

(d)

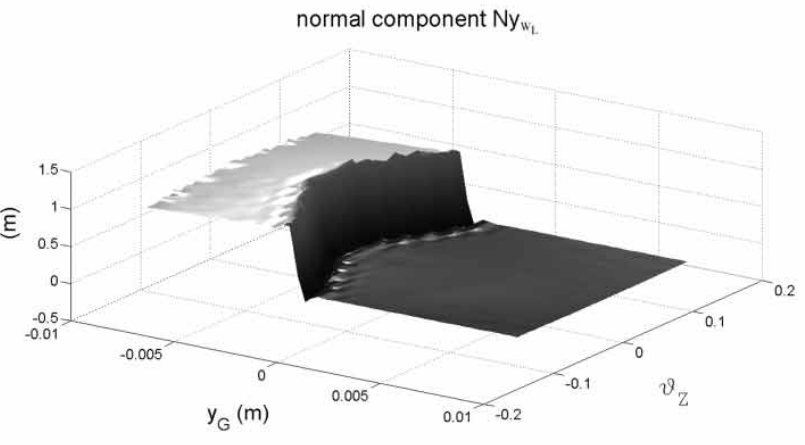

(f)

Fig. 10 Typical results stored in the lookup table. Wheel-set coordinates: (a) $z_{G}$, b) $\theta_{x}$; coordinate $z$ of the contact point on the left wheel: (c) $z$, (d) $y$; components of the normal unit vector on left wheel: (e) $z$, (f) $y$ 
Table 2 Dynamic characteristics of the wheel-set

\begin{tabular}{llll}
\hline Wheel-set mass $(\mathrm{kg})$ & 1595 & $I_{z z}\left(\mathrm{~kg} \mathrm{~m}^{2}\right)$ & 935 \\
$I_{x x}\left(\mathrm{~kg} \mathrm{~m}^{2}\right)$ & 935 & Friction & 0.2 \\
& & coefficient & \\
$I_{y y}\left(\mathrm{~kg} \mathrm{~m}^{2}\right)$ & 174 & & \\
\hline
\end{tabular}

However, in some cases a double-contact configuration occurs. In these cases, two separated volumes of intersection are generated on the same wheel. With reference to Fig. 8, two different possibilities are considered: (i) split intersecting volumes; (ii) merged intersecting volumes.

In order to establish whether the intersecting volumes are split or merged (i.e. the contact is double or single), the following test is applied: if one or more rows of aligned nodes that do not belong to $V_{\text {int }}$ exists in the group of nodes that form the surface of $V_{\mathrm{int}}$, then there are two contact points. As can be observed from Figs 3 and 8, the presence of such rows implies split intersecting volumes and double contact.

\section{GENERATION OF THE LOOKUP TABLE}

In order to reduce CPU time required, the points of contact and the normal unit vectors - for a given configuration of the wheel-set - are obtained from a previously compiled lookup table. This consists of matrices in which the dependent variables are computed as a function of the two independent variables.

The refinement adopted for the lookup table is of course a crucial topic. The chosen refinement should be found through a trade-off between precision and

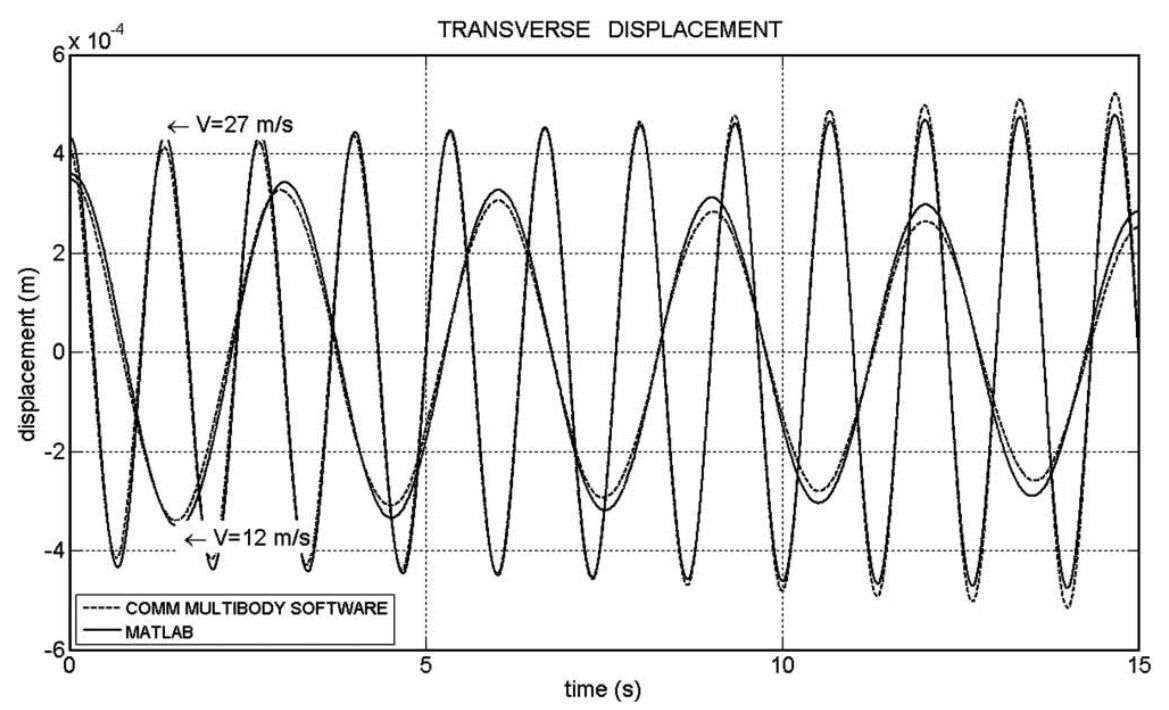

Fig. 11 Transverse displacement of the barycentre of the wheel-set; comparison between the proposed method and a commercial multi-body

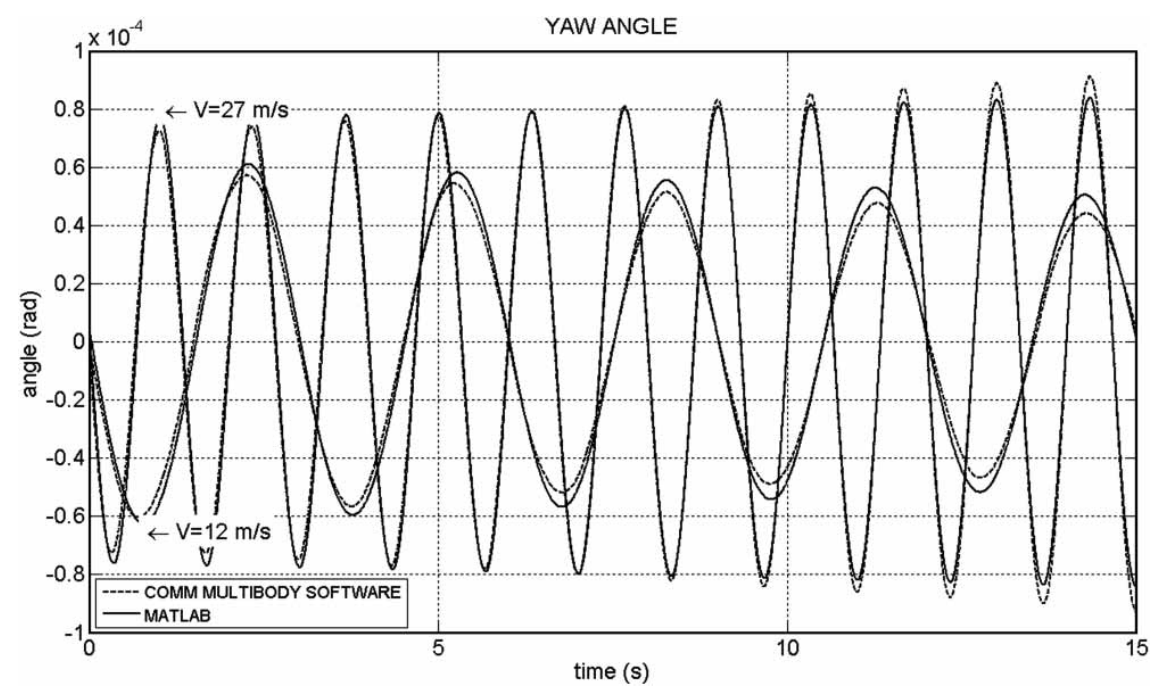

Fig. 12 Yaw angle of the wheel-set; comparison between the proposed method and a commercial multi-body 
computational costs. Thereafter, every matrix considered contains 37 rows and 37 columns both for left and right wheels. The rows represent the values of $y_{g}$, whereas the columns define the yaw angle $\vartheta_{z}$ (i.e. the two independent variables). The range of feasible equally spaced values for the independent variables is taken as follows

$$
-9 \mathrm{~mm} \leqslant y_{g} \leqslant 9 \mathrm{~mm} ; \quad-0.12 \mathrm{rad} \leqslant \vartheta_{z} \leqslant 0.12 \mathrm{rad}
$$

Given two values of the independent variables the remaining four values of the dependent variables are readily obtained through linear interpolation. Furthermore, the position of the contact points between wheels and rails and the normal unit vectors are computed making use of the interpolated data. All the stored quantities can be visualized by means of three-dimensional diagrams. The axes represent the coordinate $y_{g}$, the angle $\vartheta_{z}$, and vertical depth is the quantity to visualize. As an example, Fig. 9 shows the surface of the coordinate $x$ of the contact point on the left wheel, obtained with the three discussed methods.

As explained above, the three methods are denoted as follows: 1 (method of the centre of load), 2 (method of maximum distance), and 3 (method of maximum normal distance). One can observe the following.

1. The results of method 1 are much more smooth than the other methods; this is observed on both wheel-set positions and contact points.

2. The results of method 1 are less affected by mesh refinement.

3. Method 2 supplies slightly irregular results, mainly caused by the location of the contact points within the grids points.

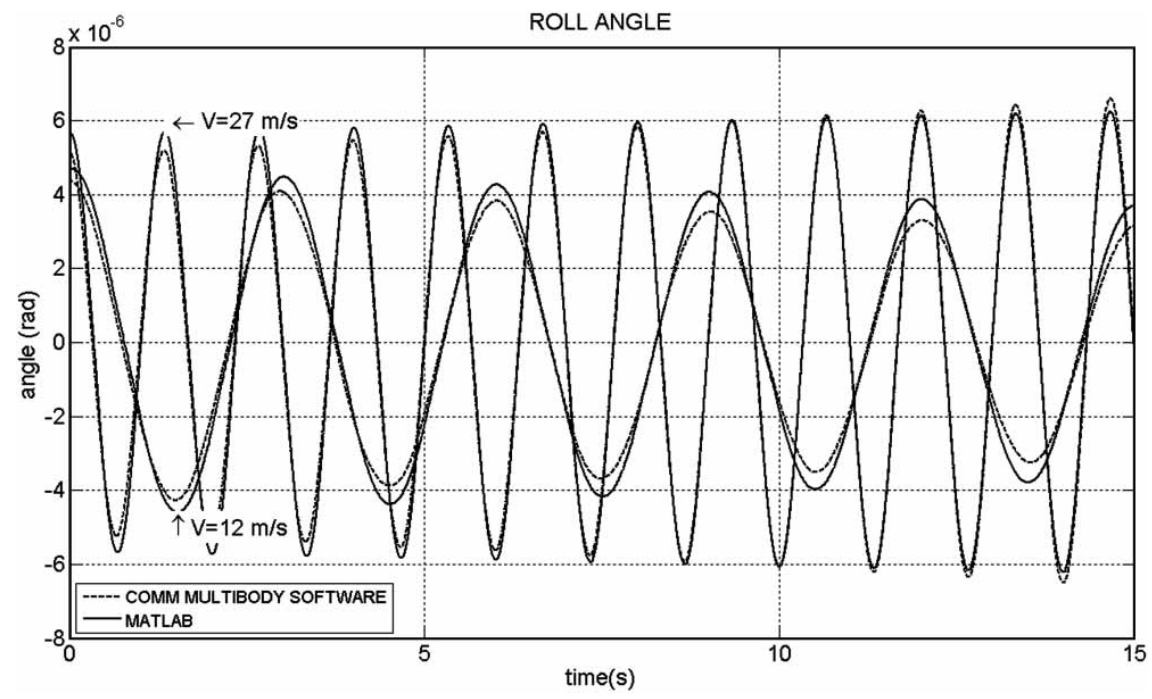

Fig. 13 Roll angle of the wheel-set; comparison between the proposed method and a commercial multi-body

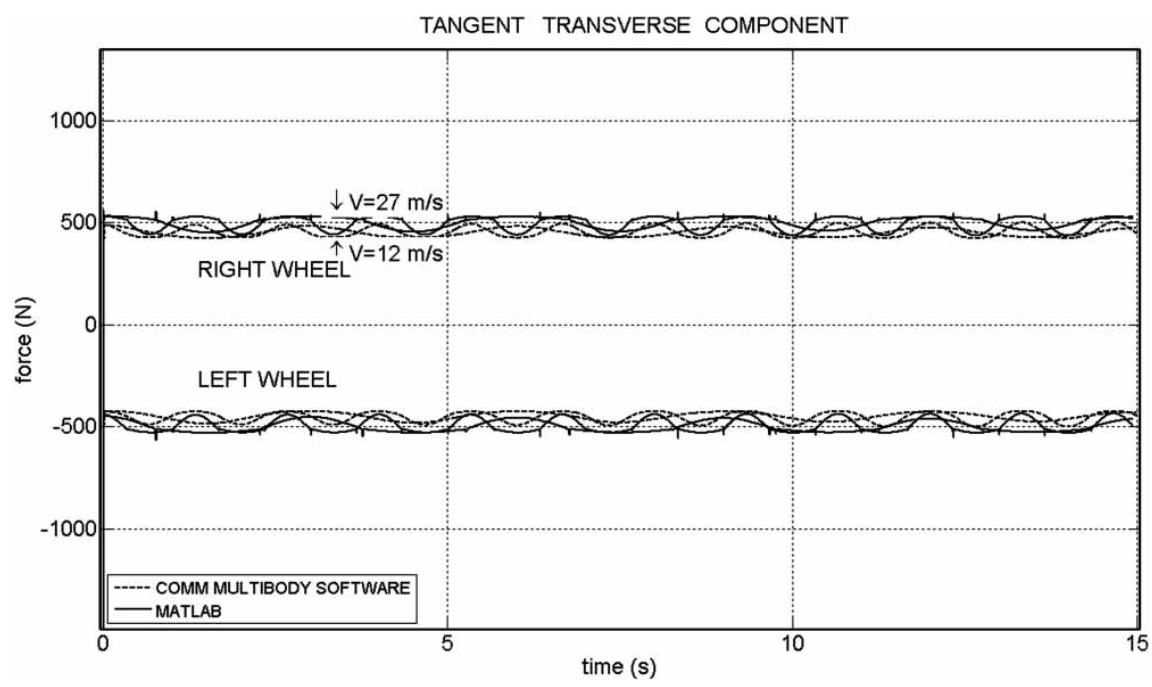

Fig. 14 Transverse component of the tangential force acting on the wheel-set; comparison between the proposed method and a commercial multi-body 
4. Method 3 supplies irregular surfaces again, although these are smoothed on the rail; this is due to the search contact algorithm that refers to wheel nodes.

In Fig. 10, the typical lookup table results are presented. In particular, in Figs 10(a) and (b) the dependent coordinate variation versus the values of $z_{g}$ and $\theta_{x}$ are shown. Since the two remaining dependent variables are very little influenced by $y_{g}$ and $\theta_{z}$, their plots are omitted. The surfaces that give the coordinates $z$ and $y$ of the contact point on the left wheel are very regular, not presenting any isolated peak as shown in Figs 10(c) and (d). Similar results are obtained for the right wheel. Finally, Figs 10(e) and (f) demonstrate that even the unit vector components, giving the attitude of the vector normals to the contact surfaces, shows a regular behaviour. In the cited figures, all the lengths are expressed in metres, whereas the angles are given in radians.

\section{VALIDATION OF THE LOOKUP TABLE IN DYNAMIC ANALYSES}

In order to validate the lookup tables, a code in Matlab environment is developed. The code is capable of simulating the motion of a dynamic system formed by one or several wheel-sets (e.g. bogie, wagon [20]).

Displacements and rotations are computed for all moving masses. When contact is experienced, the interacting force components, tangential and normal, respectively, are also computed. The tangential components are determined using Kalker's linear theory $[17,18]$ assuming a linear relation between the creepage and the tangential component. The normal component is obtained by the product of the normal indentation with the contact elastic stiffness, estimated by means of Hertz theory.

The results are compared with other performed by means of dedicated commercial multi-body software. The compared system is formed by a wheel-set resting upon rails, moving with an initial longitudinal velocity. The wheel-set is also perturbed at the initial time with a transversal velocity component equal to $0.1 \mathrm{~m} / \mathrm{s}$. A vertical force equal to $10^{5} \mathrm{~N}$ is applied on the centre of mass of the wheel-set.

The results are computed for two values of $V_{\text {long }}$ : $12 \mathrm{~m} / \mathrm{s}$ and $27 \mathrm{~m} / \mathrm{s}$. The first case concerns a stable behaviour of the wheel-set, that is to say that the longitudinal velocity is lower than the critical value $[\mathbf{5}, \mathbf{7}, \mathbf{9}, \mathbf{1 3}]$; the second computation deals with an unstable motion when the velocity is higher than the critical speed.

The dynamic characteristics of the wheel-set are reported in Table 2.

Figures 11 to 14 compare our code results with those given by the dedicated commercial software, which is also based on an off-line contact search and data extrapolation, for the following quantities:

(a) transversal displacement of the wheel-set centre of mass;

(b) wheel-set yaw and roll angle;

(c) transversal component of the tangential contact force.

The agreement confirms the reliability of the proposed method, thus validating our results at least for the wheel-set motion in both stable and unstable behaviour. In Fig. 14, it is interesting to highlight the small numerical perturbations occurring when the wheel changes its attitude. As a matter of fact, the relevant precision with which the contact is computed in the lookup table is not affected by any artificial smoothing of their values.

\section{CONCLUSIONS}

The topic of the article is the analysis of contact conditions of a wheel-set with rails. In particular, this analysis, given the two independent position variables of the wheel-set, determines the contact points on the wheel and on the rail, the relative normal unit vectors and the remaining four dependent position coordinates.

In order to reduce the computing time in a dynamic simulation of a system where several wheel-sets are present (truck or railway convoy), a lookup table created for a number of pre-established configurations is compiled. This last feature is particularly helpful for the definition of the contact conditions that can vary at each time step. Any configuration is obtained through linear interpolation of the values contained in the table. The study allows both the presence of a single contact point on the wheel, and a double contact. Under the condition of double contact, two contributions to the stiffness are included. The first one is due to the deformation of the flange, assimilated to an equivalent cantilever beam, and the second is due to the local deformation, according to Hertz's theory.

The compilation of the lookup table is carried out using three different methods. Three-dimensional plots make evident the differences between the proposed methods herein.

The method of the centre of load is by far the best since it is less sensitive to mesh refinement.

\section{(C) Authors 2011}

\section{REFERENCES}

1 Shabana, A. A., Zaazaa, K. E., and Sugiyama, H. Railroad vehicle dynamics: a computational approach, 2008 (Taylor \& Francis/CRC, Boca Raton, London, New York).

2 Sanborn, G., Tobaa, M., and Shabana, A. A. Coupling between structural deformations and wheel-rail contact 
geometry in railroad vehicle dynamics. Proc. IMechE, Part K: J. Multi-body Dynamics, 2008, 222(4), 381-392. DOI: 10.1243/14644193JMBD142.

3 Sugiyama, H., Tanii, Y., Suda, Y., Nishina, M., Komine, H., Miyamoto, T., Doi, H., and Chen, $\mathbf{H}$. Wheel/rail contact geometry on tight radius curved track: simulation and experimental validation. Multibody Syst. Dyn., 2011, 25, 117-130.

4 Lee, S. Y. and Cheng, Y. C. Hunting stability analysis of high-speed railway vehicle trucks on tangent tracks. J. Sound Vibr., 2005, 282, 881-898.

5 Knothe, K. and Böhm, F. History of stability of railway and road vehicles. In Proceedings of the 16th Symposium on Vehicle system dynamics, Technical University of Berlin, Pretoria, 1999.

6 Parena, D., Kuka, N., Vivalda, P., and Kik, W. Stability investigation and narrow curving analysis of a streetcar model. In Proceedings of the 4th Adams/Rail Users' Conference, Uthrect, 1999.

7 Banerjee, N. and Karmakar, R. Bond graph modelling of rail wheel-set on curved track. Simul. Arch., 2007, 83, 695-706.

8 Bozzone, M., Pennestrì, E., and Salvini, P. Dynamic analysis of a bogie for hunting detection considering a fast wheel-rail contact model. Multibody Syst. Dyn., 2011. DOI: $10.1007 / \mathrm{s} 11044-010-9233-8$.

9 Meli, E., Auciello, J., Malvezzi, M., Papini, S., Pugi, L., and Rindi, A. Determination of wheel rail contact points with semi analytic methods. Multibody Syst. Dyn., 2008, 20, 327-358.

10 Pombo, J. and Ambrosio, J. A. C. General spatial curve joint for rail guided vehicles: kinematics and dynamics. Multibody Syst. Dyn., 2003, 9, 237-264.

11 Pombo, J. and Ambrosio, J. A. C. Application of a wheelrail contact model dynamics to railway in small radius curved tracks. Multibody Syst. Dyn., 2008, 19, 91-114.

12 Pombo, J. and Ambrosio, J. A. C. Dynamics analysis of a railway vehicle in real operation conditions using a new wheel-rail contact detection model. Int. J. Veh. Syst. Modelling Testing, 2005, 1, 79-105.

13 Shabana, A. A., Zaazaa, K. E., Escalona, J. L., and Sany, J. R. Development of elastic force model for wheel-rail contact problems. J. Sound Vibr., 2004, 269, 295-325.

14 Shabana,A.A. and Sany, J. R. A Survey of rail vehicle track simulations and flexible multibody dynamics. Nonlinear Dyn., 2001, 26, 179-210.

15 Shabana, A. A., Tobaa, M., Sugiyama, H., and Zaazaa, K. E. On the computer formulation of the wheel/rail contact problem. Nonlinear Dyn., 2005, 40, 169-193.

16 Shabana, A. A. and Sany, J. R. An augmented formulation for mechanical systems with non-generalized coordinates: application to rigid body contact problems. Nonlinear Dyn., 2001, 24, 183-204.

17 Kalker, J. J. Three-dimensional elastic bodies in rolling contact, 1990 (Kluwer Academic Publishers, Dordrecht).

18 Kalker, J. J. Rolling contact phenomena, 2003 (Delft University of Technology, The Netherlands).

19 Meli, E., Malvezzi, M., Papini, S., Pugi, L., Rinchi, M., and Rindi, A. A railway vehicle multibody model for real-time applications. Veh. Syst. Dyn., 2008, 46(12), 1083-1105.
20 Bozzone, M., Pennestrì, E., and Salvini, P. A compliance based method for wheel-rail contact analysis. In Proceedings of the International Conference on Contact mechanics and wear of rail/wheel systems, Firenze, 15-18 September 2009.

\section{APPENDIX}

Notation
$C_{\max }^{\mathrm{L}}, C_{\max }^{\mathrm{R}}$
$E$
$\boldsymbol{F}_{\mathrm{cL}}^{h}$
$\boldsymbol{F}_{\mathrm{cR}}^{g}$
$G$
$\boldsymbol{G P}_{\mathrm{L}}^{h}$
$\boldsymbol{G P}_{\mathrm{R}}^{g}$
$\boldsymbol{H}_{i}$
$k_{\mathrm{c}}$
$k_{\mathrm{el}}^{i}$
$k_{\mathrm{rot}}^{i}$

maximum normal indentations of the left and right wheel, respectively Young's modulus contact force vector at the $h$ th contact points on the left wheel contact force vector at the $g$ th contact points on the right wheel tangential module of elasticity vectors oriented from $G$ to the $h$ th left point of contact vectors oriented from $G$ to the gth right point of contact unit vector oriented along the $i$ th axis of SRo contact stiffness stiffness of the spring acting along the $i$ th axis $(i=x, y, z)$ of SRo stiffness of the torsion spring which counteracts rotations about the $i$ th axis $(i=x, y, z)$ of SRo

$n_{\mathrm{CL}}, n_{\mathrm{CR}} \quad$ number of contact points on left and right wheel, respectively nodes number of the intersection volumes of the wheel and the rail, respectively

$N_{i}$

$\boldsymbol{N}_{j}$

$\boldsymbol{N}_{\mathrm{L}}, \boldsymbol{N}_{\mathrm{R}}$

$\boldsymbol{N}_{\mathrm{w}}$

$\boldsymbol{O P}_{\mathrm{Gw}}, \boldsymbol{O P}_{\mathrm{Gr}}$ outward unit vector normal to surface $S_{\mathrm{w}}$ at the $i$ th node outward unit vector normal to surface $\mathrm{Sr}$ at the $j$ th node outward unit vectors normal to the left and right wheel, respectively normal unit vector to the wheel surface at point $P_{\mathrm{w}}$ position vectors of the barycentre of the grid nodes on the boundaries of $V_{\text {int }}^{\mathrm{r}}$ and $V_{\text {int }}^{\mathrm{w}}$, respectively

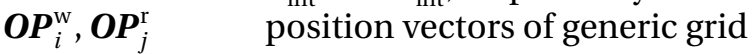
nodes on the boundaries of $V_{\mathrm{int}}^{\mathrm{r}}$ and $V_{\text {int }}^{\mathrm{w}}$, respectively

$\boldsymbol{O P}_{\mathrm{w}}, \boldsymbol{O P}_{\mathrm{r}} \quad$ position vector of the contact point on the wheel-rail computed with method of the maximum distance vector joining the nodes $i$ and $j$

Poisson's ratio 